\title{
Designing a Better Morning: A Study on Large Scale Touch Interface Design
}

\author{
Onur Asan, Mark Omernick, Dain Peer, and Enid Montague \\ Mechanical Engineering Building, 1513 University Avenue, Madison, Wisconsin, USA \\ \{asan, omernick, dpeer, emontague\}@wisc. edu
}

\begin{abstract}
In this paper, we described the design process of an individual prototype as it relates to Large Scale Public Touch Interface system design as a whole, and examine ergonomic and usability concerns for Large Scale Public Touch Interface (LSPTI) designs. The design process includes inspirational design, contextual design, storyboarding, paper prototyping, video prototyping and a user testing study. We examined the design process at each stage and proposed improvements for LSPTIs. Results indicate that the 'color-field' interaction methodology might be a good alternative to traditional 'tabbedhyperlink' interaction in LSPTI implementations.
\end{abstract}

Keywords: Intelligent space, interactive design, touch screen.

\section{Introduction}

Technology advancement allows information to be accessible from more places, thus making our life easier and more efficient. From directories in shopping malls, to weather and news in school buildings, the use of intelligent space seems to fit smoothly into our daily life. Advancement in manufacturing has reduced price of touch screens making it more cost feasible for designers to develop a product based around a Large Scale Public Touch Interface (LSPTI). LSPTIs are primarily characterized in two ways: first, in scale; while monitor-sized touch-screen installations are already part of modern installations in places like museums, LSPTIs leverage the reduced cost of touch-screen technology to create a device that takes up a large percentage of the user's field of vision (FOV). Second, the LSPTI is characterized by limited user interaction time. LSPTIs are used in indoor and outdoor settings, such as parks and museums[1]. Touch interaction allows the user to become more connected with the information he/she interacts upon, a new design dimension emerging in this era of graphic interaction [2]. Along with that, the high rate of touch screen phone use in the consumer market is a good sign of user receptiveness to interacting with Touch User Interface (TUI). Touch screen interfaces might also be a good alternative to traditional keyboards [3]. Therefore, LSPTI is a promising area awaiting more research and development. There are two design problems we would like to address in this study:

1. How should we present applications and textual data so that they would be usable by everyone?

2. What is the most efficient way to navigate through information on a Large Scale Touch Interface? 
As there is still no standard set for the large scale TUI development, we would like to put our effort in providing ground-laying recommendation on best practice in designing such Interface. To do so, our team ran various usability tests on our prototype, a mockup of a LSPTI system designed for a home bathroom setting. The device as tested had three core functions: checking weather via an internally-designed interface, checking a calendar via the Mozilla Sunbird applications, and checking email via a trimmed-down browser application.

The proposed "Better Morning" mirror introduces an easy access information spot to a bathroom. Due to the nature of bathroom, the prototype is intended for shorter face-time. In the following paragraphs, we comment on elements that assisted us in the execution of our test plan.

Magical Mirror provides simulation of interface that integrates information accessibility and basic controls on a house appliance [4]. The project introduces us to the idea of home automation [5], which forces us to define the boundary we wish to design upon. How many applications would be sufficient for a touch screen interface and what are the design implications?

Smart Mirror demonstrated implementation steps and functionality of the interactive mirror [6]. The mirror was developed as an extension to home automation system that facilitates the integration of refreshment, appliances and various customized information services.

The goal in this study is to lay the groundwork for examining the effect of ergonomic concerns on user satisfaction with LSPTI implementations and examining other key factors to LSPTI user interface design. Effective LSPTI design needs to bring together ergonomic concern, visibility, and usability into an efficient interactive experience. Every touch should result in meaningful input to the device, so presenting data in the most easily understood format is crucial.

\section{The Design Process}

The first step in the design process involved every member of the team individually completing a write-up of inspirational designs in the world around us. We found problems that society is facing today, and then found several new inventions or innovations that are helping to solve these problems. This stage, while not directly related to the final design, gave the opportunity to really break things down and learn what exactly makes a design effective and usable.

From there, the next step was looking at the user's perspective and completing a contextual inquiry. Here, we observed an eager participant performing some aspect of their daily life. This step was so useful in the design process, since it indicated that each people may perform a task or a routine in a certain way, the majority of the population often differs in their methods. This step laid the groundwork for later usertesting and stressed the importance of gathering useful data that will help improve the final design. 
"A Better Morning" as we call it, is an interactive bathroom mirror that allows users to perform several parts of their typical morning routine at the same time. It is a large touch screen interface that allows a user to perform activities such as checking the weather, checking the daily calendar, or checking email, all on a bathroom mirror. The idea behind "A Better Morning" was that it would allow the user to multitask and take the time that he or she already spends in front of a mirror, while brushing his or her teeth for instance and maximizing the value of that time by allowing the user to do much more.

The next step was to create storyboards and identify key scenarios in which this product would be used. These storyboards allowed us to get down to details and show how the product fits into a user's everyday routine, and how it will improve their quality of life.

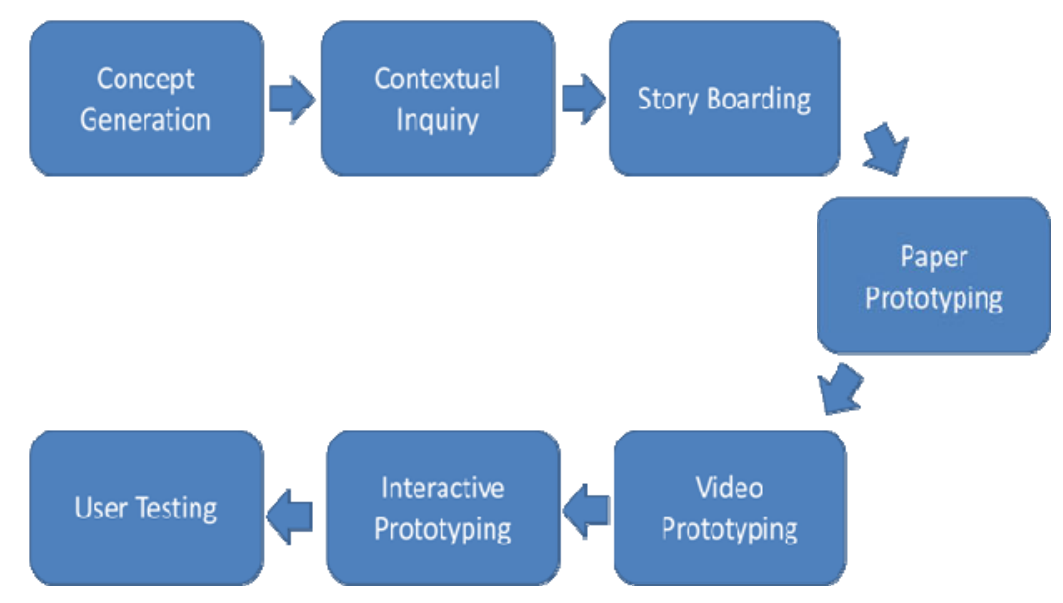

Fig. 1. Design process

\subsection{Paper Prototype}

The paper-prototype was the first real representation of the product design. We created a paper prototype that would allow participants to get a feeling for the system and interact with it in a very primitive way. This prototype was an easy way to allow us to see if our interface thus far matched a user's mental model and how we could improve it. To collect this information, we outlined three tasks for users to perform while one group member ran the prototype and the others observed. These tasks included checking the weather, checking the daily schedule, checking three separate email inboxes and responding to an email. We then had a participant complete the tasks, as we observed and took notes. The most notable thing that we learned from this step was that the method to interact with the design must match the user's mental models. 


\subsection{Video Prototype}

The next step in improving the fidelity of the prototype was to create a video prototype depicting a single user scenario. This prototype was more scripted than the paper prototype and showed less interactivity, but it did a good job of showing how the product fits into a user's daily routine, and how quality of life would be improved with this system. It portrayed a single user's morning routine without the system, and the much more streamlined routine that is made possible with the system. The video prototype allowed us to see the system in much higher fidelity and also highlighted some of the key limitations that would define the rest of the design process. We found that because of the uniqueness of our product concept, prototyping it in true form was a nontrivial task and was something that was out of the scope of the project given time constraints. We were going to have to step back and get creative to develop a way to truly test the system's interactivity in the next stage.

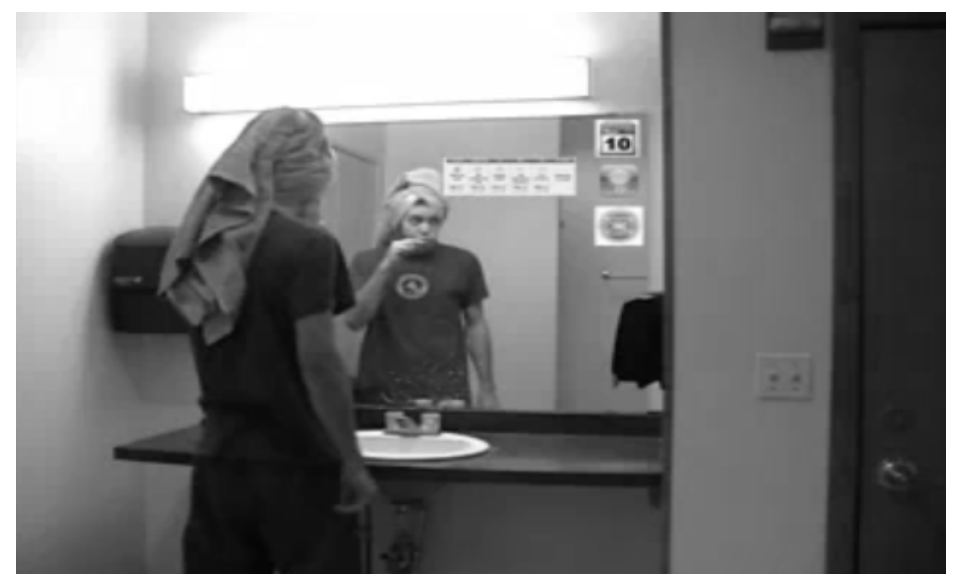

Fig. 2. Screenshot from a Better Morning Video Prototype

\subsection{Interactive Prototype}

The final step, and first real incarnation of the "A Better Morning" was the interactive prototype. This prototype consisted of an interactive PowerPoint representation of the system, projected from behind users onto a surface to mimic an actual mirror. The PowerPoint representation had all of the functionality needed to complete the tasks as previously outlined in the testing of the paper prototype. The most useful part of this prototype was the user testing that it enabled us to do. Six participants completed these tasks with the interactive prototype while we gathered data from entrance interviews, direct observations and exit interviews. This prototype was as close as we could possibly get to the actual system without physically building it. It was one of the most useful steps in the design process to this point, because of the volume and quality of feedback that were collected. 


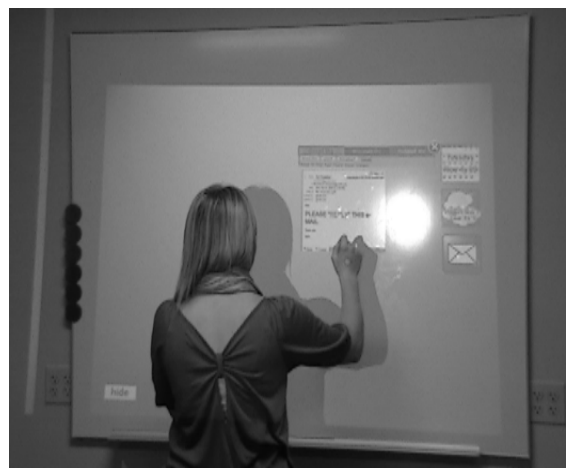

Fig. 3. Screenshot from video of A Better Morning Interactive Prototype user testing

\section{$3 \quad$ User Testing}

The user testing was conducted in a human computer interaction lab. We used a projector to act as the smart mirror on the wall. The system was established as PowerPoint, and one of the testers controlled it. When participants clicked something on the wall, it was clicked by the tester at the computer. The environment was not a real bathroom environment, but there was a preparation session for participants and they were asked to role play.

\subsection{Participants}

There were total 6 participants, three of them were female. They were all students at UW-Madison. All of the participants were frequent computer users. They all declared what kind of activities they do in the morning such as spending a significant amount of time at bathroom. Five of the participants told us that they check internet, emails or news as morning activities. We also measured the arm length and height of the participants.

Table 1. All demographic information about the participants

\begin{tabular}{llllll}
\hline Arm Length & $\#$ & Height & $\#$ & Hand & $\#$ \\
\hline 25"-29” & 3 & $5-5 ' 5$ & 2 & Right & 5 \\
30"-35" & 3 & 5'6-6 & 2 & Left & 1 \\
& & 6'1-6'5 & 2 & & \\
\hline
\end{tabular}

\subsection{Procedure}

The user testing consisted of a short pre-interview, a quantitative user observation session, and a short post-interview. During the pre-interview, one of the testers described the functionality and aim of the prototype device and collected physical 
user data (arm length and height) and qualitative data related to their routine morning activities. The user observation session consisted of three tasks, which were divided by 'simple' (requiring a minimum of $\mathrm{i} \leq 3$ interface interactions) and 'complex' (requiring a minimum of $\mathrm{i} \geq 4$ interactions). These tasks are:

1) Check today's weather. (Simple, $i=1$ )

2) Check the calendar. Examine the detail of Nov 4, which has a meeting option. Print out the detail. (Complex, $\mathrm{i}=4$ )

3) Check all of the email accounts linked to the system and respond to the email in the Gmail account. (Complex, $\mathrm{i}=6$ )

During the study, all participants were observed to collect qualitative data. These data are errors made per task, numbers of interface touches per task, completion time per task and task completion success or failure. In the post-interview, participants were asked to give feedback about the prototype. There were questions about rating their satisfaction level, ease of use for the prototype, simplicity and responsiveness of the prototype as well as their improvement suggestions for the system.

\section{$4 \quad$ Result}

All participants completed all three tasks successfully. Task completion time varies for each task and for each participant. For task 1 the average time was 14 seconds, for task 2, 35 seconds, and for task 3, 53 seconds.

We were also interested in number of screen touches used to complete each task. The following averages was obtained; task $1(\mathrm{M}=2)$, task $2(\mathrm{M}=6)$, task 3 $(\mathrm{M}=9)$.Another metric that was observed was the number of errors the participant made during the tasks. The error was defined as "any interaction with the prototype that took the user further from the interaction goal". The following numbers for each task: was obtained; task $1(\mathrm{M}=0.33)$, task $2(\mathrm{M}=1)$, task $3(\mathrm{M}=1.66)$.

After completing all tasks, participants found the product easy to use (4.83 on a scale of 1 to 5) and simple to use (4.5 on a scale of 1 to 5). The satisfaction level of participants was just below moderately satisfied (3.67 on a scale of 1 to 5).

\subsection{Usability vs. Physical Attributes}

One of the key aspects of the system examined in this prototyping session was the correlation between physical attributes (height, arm length) and core satisfaction, which was measured by the first two questions in the post-user test. The results indicate that there seems to be a correlation between height and ease of use - the only individual who did not score the system $5 / 5$ for ease of use was the shortest individual in the test study, and she further noted that the system was 'difficult to reach'. Based on this, it is possible to infer a similar correlation between ease of use and arm length, which contains a similar graph. These results indicate that the layout of the system interface presents usage challenges for individuals under 5'.

Despite the above result, there seems to be little, if any, correlation between either height or arm length and overall satisfaction, as seen below. 


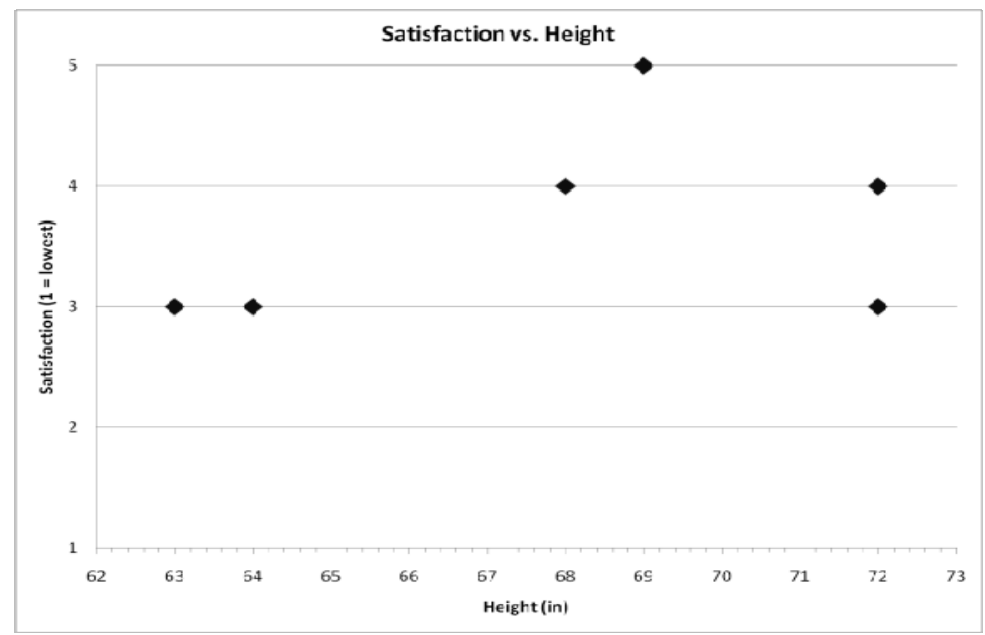

Fig. 4. Satisfaction vs. Height

These data imply other limiting factors for overall satisfaction. An examination of the exit interview free-response section reveals that $66 \%(\mathrm{M}=4)$ of users commented negatively on the size of the fonts used in the system. Furthermore, during testing, several users indicated that they could not read the fonts used. However, the smallest font used was approximately twice the size of a standard $12 \mathrm{pt}$. font read at the same distance. Since a 12-point font should be very readable , $[4,5]$ another factor must be involved.

Due to testing limitations, the projector we used was low-resolution, causing each letter to have a height of approximately 4 pixels. In addition, no antialiasing was available. Since decreasing legibility is very strongly correlated with both lack of antialiasing and decreasing the number of pixels in the letter-shape [5], the results indicate that it is not the size of the text that was the limiting factor, but rather the resolution of the display.

\subsection{Design of a Simple Interface}

Another key aspect of the system examined during this prototyping session was the simplicity of the interface. Interface simplicity was a key design goal throughout the prototyping process, and the prototype scored relatively high, marking a $4.5 / 5$ with no measured correlations. This suggests that the prototype interface represents a useful foundation for further expansion. However, the data gathered during the test has implications for the design paradigm used in LSPTI implementations.

The interface testing revealed major differences between error rate on each task. Here, error rate is defined as a user interface reaction that moves the user away from the task goal. First two tasks were easily completed, with an average of 0.5 interface errors per task. The third task, however, had significantly more errors, with an average of 1.667 interface errors per task. This suggests that the design of the interface used in the third task is significantly less efficient than those used for the first two 
The first and second interfaces are based around 'color-field' interactions, where most interactive zones are represented by either large fields of color or icons. The third interface, however, is based around 'tabbed-hypertext' interactions designed to be reminiscent of popular Internet browsers. The results suggest that the 'tabbedhypertext' design paradigm made users expect a certain set of interactions with the interface, and that the interface did not align with these expectations.

The interface testing also revealed some shortcomings in the application of the 'color-field' style interface. In the interface used for the first task, we used color fields to delineate non-interactive data areas in the display in addition to interactive zones. In the interface used in the second task, all color field areas were interactive zones. This difference resulted in a large difference in user deviation from optimal interaction paths as measured by the 'optimality ratio', or the ratio of non-optimal interactions per optimal interaction. In the first task the average optimality ratio was .83 , while in the second task the average optimality ratio was .42 , despite the second task requiring more interactions to successfully complete. This indicates that the second interface, where color fields are used exclusively to delineate interactive zones, represents a more intuitive user interface.

Table 2. Task 1, a simple task (i=1) using color-field interface

\begin{tabular}{|c|c|c|c|c|}
\hline \multicolumn{3}{|c|}{ Completion time (sec.) \# of Touches } & \multirow{2}{*}{$\begin{array}{c}\text { \# of Non-optimal touches } \\
0.83\end{array}$} & \multirow{2}{*}{$\begin{array}{c}\text { \# of Errors } \\
0.5\end{array}$} \\
\hline Mean & 14.33 & 1.83 & & \\
\hline S.D & 8.80 & 0.75 & 0.75 & 0.55 \\
\hline
\end{tabular}

Table 3. Task 2, a complex task (i=4) using color-field interface

\begin{tabular}{|c|c|c|c|c|}
\hline \multicolumn{3}{|c|}{ Completion time (sec.) \# of Touches } & \multirow{2}{*}{$\begin{array}{c}\text { \# of Non-optimal touches } \\
1.67\end{array}$} & \multirow{2}{*}{$\begin{array}{c}\text { \# of Errors } \\
0.5\end{array}$} \\
\hline Mean & 35.33 & 5.67 & & \\
\hline S.D & 6.62 & 1.03 & 1.03 & 0.55 \\
\hline
\end{tabular}

Table 4. Task 3, a complex task (i=6) using a tabbed-hyperlinked interface

\begin{tabular}{|c|c|c|c|c|}
\hline \multicolumn{3}{|c|}{ Completion time (sec.) \# of Touches } & \multirow{2}{*}{$\begin{array}{c}\text { \# of Non-optimal touches } \\
3.33\end{array}$} & \multirow{2}{*}{$\begin{array}{r}\text { \# of Errors } \\
1.7\end{array}$} \\
\hline Mean & 55.67 & 9.33 & & \\
\hline S.D & 13.85 & 1.37 & 1.37 & 0.82 \\
\hline
\end{tabular}

\section{Discussion}

This study has revealed several areas for improvement in the design of LSPTI. First, we found that an LSPTI should be able to adjust to the reach ability of the user, in 
order to avoid placing interactive zones outside of the user's reachable area. While this would not normally cause a concern for smaller TUI applications, the inherent larger size of LSPTI installations requires this adjustment. The results suggest that this is one of the main causes of decreased ease of use. To accomplish this, either some sort of interface control could be created that would allow a user to 'drag' the interface down to a reachable level, or perhaps some sort of spatial-recognition feature could be integrated into LSPTI-based devices which would be able to ascertain the rough physical dimensions of the user.

Second, we found that high-resolution displays are very important for future LSPTI implementation. While a low-resolution projector may be appropriate for presenting at a distance, they are not as useful when a user is within arm's length of a screen. At 20-30 range, the amount of data that can be discerned on a large, low-resolution device is very low, and can cause user errors and dissatisfaction.

Third, we found that the current model of 'tabbed-hypertext' browser interactions does not serve the design of LSPTI well, allowing more user errors than the proposed 'color-field' interaction methodology. 'Color-field' takes better advantage of the larger space available on LSPTI implementation and allows users to interact with larger active areas, ultimately reducing the number of interface errors. Given that LSPTI installations may be found in areas where the user would have a limited ability to use the interface to the point where he or she could develop a strong mental model, reducing interface complexity is the key factor.

Finally, we found a key limitation of the 'color-field' interaction methodology. If a designer uses 'color-field' in a LSPTI implementation, there must be a clear distinction between which areas are interactive zones and which areas are noninteractive, used only to delineate different data areas. Failure to do this will lead to user confusion and inefficient interface traversal.

\section{$6 \quad$ Future Study}

There is a great deal of future work to be done in the LSPTI field. The wide range of additional applications suggested by our participants indicates that LSPTI-based interfaces have a wide potential for further development. The preliminary results encourage further studies in the areas of 'color-field' interaction methodology as an alternative to traditional 'tabbed-hyperlink' interactions in LSPTI implementations.

\section{References}

1. Nakanishi, H., Koizumi, S., Ishida, T., Ito, H.: Transcendent communication: location-based guidance for large-scale public spaces. In: Conference Transcendent Communication: Location-based Guidance for Large-scale Public Spaces, p. 662. ACM, New York (2004)

2. Panchanathan, S., Kahol, K.: Guest Editors' Introduction: Haptic User Interfaces for Multimedia Systems. In: IEEE Multimedia, pp. 22-23 (2006) 
3. Plaisant, C., Sears, A.: Touchscreen interfaces for alphanumeric data entry. In: Conference Touchscreen Interfaces for Alphanumeric Data Entry, pp. 293-297. Human Factors and Ergonomics Society (1992)

4. Tinker, M.: Legibility of Print for Children in the Upper Grades. American Journal of Optometry and Archives of American Academy of Optometry 40, 614 (1963)

5. Sheedy, J., Subbaram, M., Zimmerman, A., Hayes, J.: Text legibility and the letter superiority effect. Human Factors 47, 797 (2005)

6. Hossain, M., Atrey, P., El Saddik, A.: Smart mirror for ambient home environment. In: Conference Smart Mirror for Ambient Home Environment, pp. 24-25 (2007) 